\title{
Correction to: Marcel Grossmann
}

\section{Correction to:}

C. Graf-Grossmann, Marcel Grossmann, Springer Biographies, https://doi.org/10.1007/978-3-319-90077-3

The original version of the book was inadvertently published without the following corrections in frontmatter:

A blank page should be included after family tree.

Copyright information text should be updated.

Translator (William D. Brewer) affiliation should be added.

The correction book has been now updated with the changes. 